amplitude leads, QTc interval prolongations, Left ventricular hypertrophy. The coronary arteries on complete close up anterior wall or complicated anterior more control of wall AMI and LAD merge LCX or RCA was significantly increased $(p<0.05-0.01)$. Clinically occurrence of complications such as pump failure, infarct extension, angina pectoris, malignant arrhy mortality ventricular electrical storm $6 \mathrm{~h}$ after AMI was obviously increased $(\mathrm{p}<0.05-0.01)$.

Conclusion For those after hypertrophy and AMI patients with VES who having abnormal ECG indexes, clinical shortterm prognosis was poor. Comprehensive measures should be taken to improve prognosis.

\section{e0582 THE DETECTION OF CAROTID ARTERY INTIMA PLAQUE IN ELDERLY HOSPITALISED PATIENTS WITH HYPERTENSION AND ITS INFLUENCING FACTORS}

doi:10.1136/hrt.2010.208967.582

${ }^{1}$ Sheng Hao LIU, 'Wen Bo PAN, ${ }^{1}$ Wan Sheng ZHONG, ${ }^{2}$ WANG Ying. ${ }^{1}$ Department of Cardiovascular Medicine, Hefei Third People's Hospital, Hefei, China; ${ }^{2}$ Department of Colour Doppler Ultrasonic, Hefei Third People's Hospital, Hefei, China

Objective To study the detection of carotid artery intima plaque in elderly hospitalised patients with hypertension and its influencing factors.

Methods Selected my hospital in the entire group of elderly hypertensive patients with a total of 328, used colour Doppler ultrasound to detect all patients' carotid artery intima plaque and grading. Patients' sex, age, the course of hyperpiesia, the controls of high blood pressure in the past, the smoking history, family history of hypertension, history of stroke and other messages were noted by clinical records.

Results The detection rate of carotid artery intima plaque was $77.4 \%$. In which Class 1 , Class 2 and 3 accounted for $32.0 \%, 36.9 \%$ and $8.5 \%$ (plague score). Use Ordered Logistic regression univariate cumulative analysis to show that male, old, poorly controlled blood pressure, longer duration of hypertension were risk factors for plaque. Multivariate analysis showed that the major risk factors for plaque were male, old age and poor control of blood pressure.

Conclusions Hypertension in the elderly patients with higher rate of carotid artery intima plaque, and male patients, old age, patients whose blood pressure was in poor control were the high-risk groups.

\section{e0583 THE INTERVENTION ROLE OF VALSARTAN ON LEFT VENTRICULAR HYPERTROPHY AND HIGH-SENSITIVITY C-REACTIVE PROTEIN I N HYPERTENSION PATIENTS}

doi:10.1136/hrt.2010.208967.583

Cen Oiang, Yang Ming, Zhao Yan. Fuxing Hospital Affiliated Capital Medical Univercity/ ccu

Objective To explore the relationship between high-sensitivity Creactive protein (hs-CRP) and left ventricular hypertrophy (LVH) and to examine whether angiotensin receptors antagonist ARB could decrease CRP expression, block or invert the progression of LVH with essential hypertension (EH).

Methods $186 \mathrm{EH}$ patients were divided into LVH group $(\mathrm{n}=92)$ and non-LVH group $(\mathrm{n}=94)$ by Devereux standard. All patients received Valsartan therapy for $24 \mathrm{w}$ t o detect blood pressure, hs-CRP and left ventricularmass index (LVM I) 1

Results The hs-CRP level of LVH group was higher than that of non-LVH group. Hs-CRP was positively correlated with LVM I. HsCRP decreased obviously in non- LVH and LVH group s after treat ment. LVM I decreased significantly than before treatment in LVH group $(p<0.01)$.
Conclusions Plasma hs-CRP level is an independent risk factor for the development of LVH in EH. Valsartan treatment can decrease the incident $\mathrm{LVH}$ by reducing hs-CRP.

\section{e0584 A STUDY OF EXFORGE ON THE TREATMENT EFFECTS AND CVE PREVENTION IN PATIENTS WITH MILD TO MODERATE ESSENTIAL HYPERTENSION}

doi:10.1136/hrt.2010.208967.584

Zhang Ying, Zhou Litao. The Public Health Department of Zhejiang University

Objective To compare the difference of the treatment effects and the incidence of CVE in further 10 years in the mild to moderate essential hypertensive population who used Exforge-a new fixed combination regimen, or Norvasc-a classic antihypertensive monotherapy, in order to provide more choices of regimens for them.

Designs and Methods This stage III clinical trial was 8-week and randomised. Patients received Exforge (Amlodipine $5 \mathrm{mg} /$ Valsartan $80 \mathrm{mg}$ ) or Norvasc (Amlodipine $5 \mathrm{mg}$ ) once daily. The difference of blood pressure reductions between them were compared. The Chinese Multi-Provincial Cohort Study (CMCS) model, which is an adjusted model of Framingham model in China, was used to predict the incidence of CVE in further 10 years before and after taking the drug regimens in hypertensive patients with different levels of risk factors. Results A total of 256 patients were randomised to the treatment groups (128 in Exforge group and 128 in Norvasc group), and the overall mean age was 54.4 years. (1) The treatment effects in Exforge group was better than that in Norvasc group, and the blood pressure lowering efficiency were $84.9 \%$ and $71.9 \%$ respectively. Meanwhile the risk difference was 13.0\% (12.49\%-13.51\%), which was statistically different $(\chi 2=6.692, p=0.010)$. (2) After taking the anti-hypertensive treatment, the incidence of CVE in further 10 years were smaller than those before therapy, and the reduction of incidence in Exforge group were more than those in Norvasc group. The preventive effects on stroke were more obvious than those on CHD. 1. Before taking the hypertensive treatment, the incidence of $\mathrm{CHD}$ in further 10 years was $17.40 \%$ at the baseline level of risk factors, and after the treatment, the incidence were $9.43 \%$ in Exforge group and $12.22 \%$ in Norvasc group, reducing $45.81 \%$ and $29.74 \%$ respectively. The reduction of incidence in Exforge group was 1.54 times than that in Norvasc group. Before taking the hypertensive treatment, the mean incidence of $\mathrm{CHD}$ in further 10 years was $27.63 \%$ at different levels of risk factors, and after the treatment, the mean incidence were $15.74 \%$ in Exforge group and $19.53 \%$ in Norvasc group, reducing $44.44 \%$ and $29.93 \%$ respectively. The reduction of incidence in Exforge group was 1.48 times than that in Norvasc group. 2. Before taking the hypertensive treatment, the incidence of stroke in further 10 years was $24.51 \%$ at the baseline level of risk factors, and after the treatment, the incidence were $8.31 \%$ in Exforge group and $15.22 \%$ in Norvasc group, reducing $66.82 \%$ and $37.91 \%$ respectively. The reduction of incidence in Exforge group was 1.76 times than that in Norvasc group. Before taking the hypertensive treatment, the mean incidence of stroke in further 10 years was $33.81 \%$ at different levels of risk factors, and after the treatment, the mean incidence were $12.71 \%$ in Exforge group and $22.34 \%$ in Norvasc group, reducing $63.02 \%$ and $34.60 \%$ respectively. The reduction of incidence in Exforge group was 1.82 times than that in Norvasc group.

Conclusions Compared to Norvasc, Exforge had better treatment effects in the patients with mild to moderate essential hypertension. The reductions of incidence of CVE in future 10 years in Exforge group were more than those in Norvasc group, and the preventive effects on stroke were more obvious than those on CHD. Exforge could be recommended to use in anti-hypertensive treatment and the CVE prevention. 